\title{
Knee joint preservation: a call for daily practice revival of realignment surgery and osteotomies around the knee
}

\author{
Ronald J. van Heerwaarden ${ }^{1}$ Michael T. Hirschmann ${ }^{2}$
}

Received: 22 August 2017 / Accepted: 24 August 2017 / Published online: 15 September 2017

(c) European Society of Sports Traumatology, Knee Surgery, Arthroscopy (ESSKA) 2017

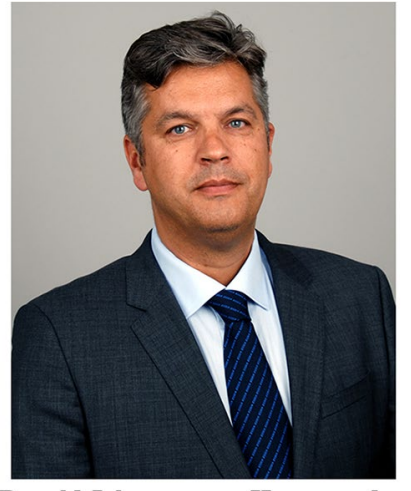

Ronald Johannes van Heerwaarden

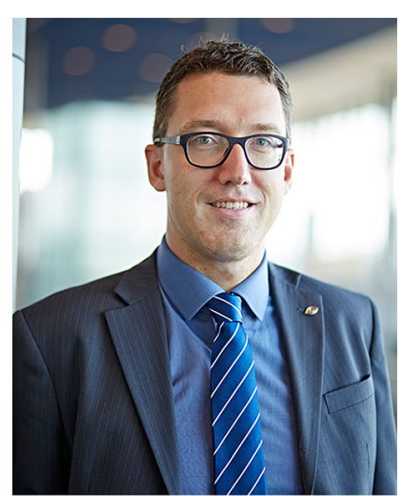

Michael Tobias Hirschmann
For joint preserving treatment of the degenerative knee, achieving a balanced mechanical leg axis is one of the most important goals. A previously published treatment algorithm has given osteotomies highest priority [1]. The hierarchy of treatment should be alignment, stability, meniscus and finally cartilage surgery.

Having gained experience in centres with extensive knowledge on joint preserving therapy leads the authors to state that a re-focus on leg alignment is necessary. In a time in which medical education for knee surgeons becomes increasingly specialized, it is even more important to see the

Ronald J. van Heerwaarden vanheerwaarden@yahoo.com

1 Centre for Deformity Correction and Joint Preserving Surgery, Kliniek ViaSana, Hoogveldseweg 1, 5451 AA Mill, The Netherlands

2 Department of Orthopaedic Surgery and Traumatology, Kantonsspital Baselland (Bruderholz Liestal, Laufen), 4101 Bruderholz, Switzerland knee as an organ and consider the whole picture of treatment. Clearly, in cases in which leg realignment was not performed as a first step of treatment, experience painfully taught us a lesson that other well-intended joint preserving treatments like ligament reconstructions, meniscal repairs or replacement procedures as well as cartilage repair are often doomed to fail. Still many surgeons, experts in their own subspecialized field of knee surgery have not incorporated this algorithm in their daily practice causing well-intended treatments to fail. A reason for missing this important link may be the way we learn and incorporate knowledge into our daily practice.

Bruce Reider [2] once wrote that physicians after their training enter into a lifelong career of learning and relearning. Keeping up with all new information, choose what is worthwhile for one's own practice, and then incorporate this into daily practice, however, is not an easy task. Knowledge acquisition and implementation has become a scientific study by itself not in the last place because of the huge amount of information that can be obtained in our fields of specialization. To make life easier, one can follow widely available courses on information management, literature search strategies and health care statistics.

Since childhood, knowledge has been presented to us by parents or peers, skills were developed by practicing and repetition, and skills were mastered by updating and further learning. Translated to our daily practice, this would mean: (a) remember what our teachers told us; (b) read textbooks and articles specific to the subject; (c) visit meetings, specialty sessions, practical teaching courses with hands-on practicing or visit expert centres and work with experienced colleagues, and then (d) incorporate the skills in your treatment armamentarium. The final step is (e) to update your knowledge by reading the literature and visit meetings where the latest knowledge is discussed. 
If we apply these principles, we should remember what our peers have told us, who have been raised and working in a time in which osteotomy surgery was a commonly used standard procedure. Most of our European godfathers of knee surgery such as Henri Dejour, René Marti, Werner Müller, and Giancarlo Puddu were also masters in osteotomy. During their lifelong careers, they have shown that after state of the art corrective osteotomies progression of osteoarthritis is delayed or even stopped [3-6]. In 2013, the KSSTA journal devoted the January issue to the subject of osteotomies around the knee to focus on the rapid evolution of osteotomy surgery due to modern osteotomy concepts, new and safe surgical techniques and better fixation methods.

In its courses, ESSKA has been one of few international knee societies focusing on knee joint preserving therapy including osteotomies. Through the years, many specialty sessions on osteotomies around the knee have been held at their biannual meetings and, by invitation, as ESSKA sessions at other knee society meetings. Hence, it is only logical that in 2016 ESSKA established the Osteotomy committee constituting of 15 European osteotomy experts as a focus group of its European Knee Associates (EKA) section.

Worldwide a growing number of courses offer practical teaching on realignment surgery with hands-on exercises in osteotomy planning, and practical exercises on sawbones and wet-lab human specimens. The ESSKA Osteotomy committee is at the moment working on a roadmap of the different courses held in Europe and will present a module-based certification system for these courses in the near future. Live-surgery videos of standard osteotomy surgery and presentations of osteotomy experts can be found on the ESSKA Academy website. In addition, ESSKA organizes two specific fellowships for young surgeons eager to visit knee experts: the ESSKA Arthrex Osteotomy travelling fellowship offering visits to osteotomy expert centres in Europe and the ESSKA-EKA European Arthroplasty travelling fellowship in which osteotomy surgery is also demonstrated by expert knee surgeons. Another option is to visit colleagues specialized in realignment surgery or apply for visiting programs or fellowships offered at their hospitals. Based on the aforementioned a knee surgeon interested in osteotomy surgery has a variety of different learning options to grow his skills to a level where it is possible to safely use them in daily practice.

Finally, already acquired knowledge can be updated by visiting meetings such as next year's ESSKA biannual meeting in Glasgow, in which two instructional courses on osteotomies will be held.

This present KSSTA issue also contains a special section on osteotomies. In the opening article, Madry et al. remind us of one of the first principles we have learned: first do no harm. Their description of anatomic structures at risk, related to the opening wedge high tibial osteotomy procedure, should be incorporated in every knee surgeon's knowledge. In the second article, Lansdaal et al. have proven the safety of an immediate weight bearing protocol tested in a randomized controlled trial of patients treated with an opening wedge high tibial osteotomy utilizing a locking plate without bone grafting. In their discussion of this well-performed study, the authors highlight the importance of educating physiotherapists in the optimal rehabilitation protocols.

The other articles in this issue might further complement and extend your knowledge in osteotomy surgery. Please enjoy reading the articles published in this issue and try to incorporate the new knowledge in your daily practice. Learning options on the subject of joint preservation and osteotomies are numerous, and ESSKA is very active in offering these options. Finally, we hope that this editorial might motivate you to see the whole picture of knee surgery. For the sake of your patients always think about leg realignment surgery when you are involved in knee joint preserving treatments.

\section{Compliance with ethical standards}

Conflict of interest Ronald Johannes van Heerwaarden and Michael Tobias Hirschmann declare that they have no conflict of interest.

Funding No funding was received for this editorial.

Ethical approval All procedures performed in studies involving human participants were in accordance with the ethical standards of the institutional and/or national research committee and with the 1964 Helsinki declaration and its later amendments or comparable ethical standards.

Informed consent Formal consent is not required for the editorial.

\section{References}

1. Arnold MP, Hirschmann MT, Verdonk P (2012) See the whole picture: knee preserving therapy needs more than surface repair. Knee Surg Sports Traumatol Arthrosc 20:195-196. doi:10.1007/ s00167-011-1733-4

2. Reider B (2010) Lifelong learning. Am J Sports Med 38(2):229_ 230. doi:10.1177/0363546509360181

3. Dejour H, Walch G, Deschamps G, Chambat P (1987) Arthrose du genou sur laxité chronique antérieure. Rev Chir Orthop Reparatrice Appar Mot 73(3):157-170

4. Marti RK, Van Heerwaarden RJ (eds) (2008) Osteotomies for posttraumatic deformities. Georg Thieme Verlag, Stuttgart, New York

5. Muller W (2001) High tibial osteotomy. Eur Instr Course Lect 5:194-206

6. Puddu G, Franco V, Cippola M, Cerullo G, Gianni E (2008) Opening wedge-proximal tibia and distal femur. In: Jackson DW (ed) Reconstructive knee surgery master techniques in orthopaedic surgery, 3rd edn. Lippincott Williams \& Wilkins, Philadelphia, pp 433-450 